\title{
Spectral Re-Growth Suppression in the FBMC- OQAM Signal Under the Non-linear Behavior of a Power Amplifier
}

\author{
Jimmy Jake \\ Department of Electrical Engineering, \\ Pan African University, Institute for \\ Basic Sciences, Technology and \\ Innovation, Nairobi, Kenya \\ jakeloponi@gmail.com
}

\author{
Elijah Mwangi \\ School of Engineering, \\ University of Nairobi, \\ Nairobi, Kenya \\ mwangiel2010@gmail.com
}

\author{
Kibet Langat \\ Dpt of Telecommunication and \\ Information Engineering, Jomo Kenyatta \\ University of Agriculture and \\ Technology, Nairobi, Kenya \\ kibetlp@jkuat.ac.ke
}

\begin{abstract}
Typically, the filter bank multicarrier with offset quadrature amplitude modulation (FBMC-OQAM) bears some impressive properties that make it popular as one of the substitutes to orthogonal frequency division multiplexing (OFDM) for the upcoming technology of broadband wireless communication systems. Although FBMC-OQAM preserves the multicarrier modulation (MCM) features, its spectrum usually suffers from impairments when subjected to the nonlinear behavior of a power amplifier (PA) which results in spectral regrowth. Due to the spectrum limitation and low energy efficiency foreseen in the forthcoming $5 \mathrm{G}$ networks, it is vital to confine the spectrum of the FBMC-OQAM signal within the allocated band of interest. In this paper, the suppression of the spectral regrowth experienced on the FBMC-OQAM signal due to the nonlinear distortion effects introduced by the $P A$ is investigated. The crest factor reduction (CFR) method in combination with an adaptive digital pre-distortion (DPD) are used. The peak windowing technique based on sequential asymmetric superposition windowing (SASW) algorithm is used in the CFR part while the least square estimation with QR-decomposition (LSE/QR) has been used as the coefficient's estimator and adaptation algorithm in the DPD part. The performance of the two combined techniques has been evaluated on SystemVue2018 simulation platform. The adjacent channel leakage ratio (ACLR) and the error vector magnitude (EVM) have been considered as the performance merits. The simulation results show that the proposed techniques significantly improve the spectrum, first by reducing the PAPR of the FBMC-OQAM signal by about $1.5 \mathrm{~dB}$. Secondly, the spectral re-growth has been reduced by about $-45.74 \mathrm{~dB}$ adjacent channel leakage suppression and the error vector magnitude measure has been obtained to be about $7.12 \%$. $(-22.95 \mathrm{~dB})$. These values lead to better average input power of the FBMC-OQAM signal and improvement in the spectral efficiency and they are in accordance with the 3GPP standard for wideband signals in nonlinear systems.
\end{abstract}

Keywords-FBMC-OQAM; spectral re-growth; nonlinear PA; CFR; SASW; adaptive DPD; LSE with QR decomposition

\section{INTRODUCTION}

The dynamic growth in the number of users that have different demands for accessing information under wireless communication is leading to spectrum scarcity. This has prompted a concern for the investigation of new techniques to address the issues of spectrum and energy efficiency improvement. Orthogonal frequency division multiplexing (OFDM) modulation scheme had been widely used in the existing wireless domain due to its ability to effectively minimize the issues of delay spread in the broadband wireless channels in the long-term evolution (LTE) 4G cellular networks [1-3]. However, the OFDM signal is time-limited because it is centered on the use of rectangular pulses in the time domain which leads to slowly decaying behavior in the frequency domain. These features make OFDM inappropriate, especially in situations where users need to be asynchronized and strict limits on the out-of-band radiation levels are highly required.

To overcome these limitations, the FBMC-OQAM scheme has been studied as an alternative waveform candidate to OFDM and is becoming the leading contender among the multicarrier modulation schemes proposed for the upcoming wireless communication systems, because of its ability of minimizing the out-of-band emission (OOBE), cyclic prefix (CP) free transmission, and robustness to the asynchronous situation [4-6]. Unfortunately, the FBMC-OQAM signal still suffers from high peak-to-average power ratio (PAPR) which is susceptive to the nonlinear behavior of the PA, and as a result, it suffers from spectral re-growth. High peak signals require amplification with power amplifiers that have high linearity. However, such power amplifiers come with high cost and they compromise energy efficiency $[7,8]$ which may not be suitable to many telecommunication network providers, that is why nonlinear power amplifiers are still common in many wireless transmitters, due to the high cost and low energy efficiency of the linear power amplifiers, the use of nonlinear power amplifiers is preferred. However, they cause signal distortion which requires compensation techniques for signals with high peak values. Among these techniques are clipping and filtering, peak cancellation, and peak windowing for crest factor reduction [9, 10] while feedforward, feedback, and predistortion $[10,11]$ were used for nonlinearity compensation. 
These techniques allow the nonlinear PA to operate at higher output power while maintaining linearity and increase in energy efficiency. Reducing the crest factor enhances energy efficiency and linearization approaches required to compensate the nonlinearities introduced by the PAs. However, in practice, the crest factor reduction (CFR) technique slightly degrades the signal quality which results in reduced data throughput. In regard to multicarrier signals such as FBMC-OQAM, degradation within the required bandwidth has less significance than degradation that results from the broadening of signal bandwidth [10].

In this work, the peak windowing approach $[9,10]$ based on the Kaiser window together with sequential asymmetric superposition widowing (SASW) algorithm [12] is used to reduce the high peak values experienced on the FBMC-OQAM signal. The reasons for this are that this algorithm is applicable to both single and multicarrier signals. Also, it can handle the over-attenuation issues that arise as a result of unnecessary window superpositioning. The concurrent application of crest factor reduction and digital pre-distortion techniques can provide an amicable solution to meet the requirements of linearity and energy efficiency benefits [13]. Digital predistortion (DPD) techniques are currently popular in the cellular communication domain as nonlinearity compensating techniques [14]. Due to the high flexibility and excellent linearization performance, DPD has been mostly used in linearizing PAs and it tends to be an essential linearization technique in the current and next-generation wireless communication systems. Owing to the dynamic nature of the PA nonlinearity, adaptive digital pre-distortion will be used here as a nonlinearity compensating technique to deal with the issues of the spectral re-growth experienced on the FBMCOQAM signal in order to maintain the characteristics of the signal under the nonlinear behavior of the PA. Although adaptive digital pre-distortion can be modeled as a nonlinear technique using Volterra Series, Look-up Table (LUT) and Memory Polynomial (MP) $[15,16]$, the main focus here is on the memory polynomial model which is a truncated version of Volterra series due to its elegance and simplicity in implementation. The least square estimation (LSE) algorithm has been used on many occasions to estimate the coefficients of nonlinear systems $[10,17]$. Here, the LSE with QR decomposition is used to estimate and adapt the model's coefficients. This is because of its ability in dealing with the over-determined problems which may arise because of the large number of samples used and also the ill-conditioned and rank-deficient scenarios that may be encountered during the inversion of the transfer function of the nonlinear power amplifier.

To the best of our knowledge, no published study has used the combination of the two algorithms to concurrently minimize both the PAPR and the spectral re-growth of the FBMC-OQAM under the nonlinear effects of the PA.

\section{RELATED WORK}

In [15], the impact of HPA nonlinearity on the performance of OFDM and FBMC-OQAM systems was investigated, where Saleh's model for the nonlinear HPA was considered. In this study, two pre-distortion schemes which were based on the indirect learning architecture were presented. The first scheme aimed to compensate simultaneously the amplitude and phase distortions induced by the nonlinear HPA, while the second aimed to compensate these distortions separately. It was shown that the first pre-distortion scheme performs worse in the FBMC-OQAM system than in the OFDM. With the second pre-distortion scheme, the phase and amplitude pre-distortions were made separately. The OFDM and FBMC-OQAM systems reached the same performance showing that a higher attention must be paid in phase correction in FBMC-OQAM.

Authors in [18] presented an innovative algorithm for scalar feedback digital pre-distortion, known as orthogonal scalar feedback linearization to compensate the nonlinear distortion and reduce the spectral re-growth in nonlinear power amplifiers. The adaptation of the discrete model coefficients becomes orthogonal in the intermodulation domain. This scheme presented a lower intermodulation at the power amplifier output and was compared with existing scalar feedback digital pre-distortion algorithms in achieving faster convergence time and lower output power variation. The problem of this method is that the coefficients are adjusted independently while the lower order coefficients are affected by the higher order ones. Also, the adjustment on the current coefficient affects the adjustments made on the previous coefficients, which causes non-orthogonality of coefficients in the intermodulation domain. The distortion caused by nonlinear power amplifiers brought up the desire for correction. In [19], the performance of $5 \mathrm{G}$ candidate waveforms such as FBMC and UFMC with nonlinear power amplifiers was evaluated using digital pre-distortion and an iterative correction algorithm with hard detection and was compared to the OFDM waveform. It was observed that, in regards to power amplifier without memory effects, the UFMC presents the most robust behavior while the FBMC suffers more impact in terms of BER. It was also noted that the iterative correction with hard detection algorithm is effective on the three waveforms and that no many iterations are needed to reach a result close to linear performance. The scenario with memory power amplifier changes this result a bit. In this case, the FBMC is the one that overcomes the others in terms of BER performance. The $5 \mathrm{G}$ waveforms had showed more robustness when compared to the 4G waveform (OFDM).

\section{SYSTEM MODEL}

\section{A. Overview of the FBMC-OQAM System}

The overall concept of the FBMC-OQAM is the transmission of complex symbols, where the in-phase and quadrature components are interleaved by a half symbol duration, $T / 2$. In the OQAM pre-processing, the complex input symbol vectors $C_{m, q}$ are converted into real symbols, where the in-phase and the quadrature components are time staggered by half a symbol period in order to maintain the orthogonality between carriers. For $0 \leq m \leq 2 M-1$ and $0 \leq n \leq N-1$, where $m$ and $n$ are the symbols and subcarriers indices respectively, the OQAM processing mathematical formulation is given as in [20]. The procedure of the OQAM pre-processing in the FBMC transmitter is illustrated as in Figure 1. After the OQAM preprocessing, the real symbols undergo poly-phase filtering that 
involves IFFT transformations along with filtering by a synthesis filter bank (SFB) with an impulse response $g(k)$ as illustrated in Figure 2.

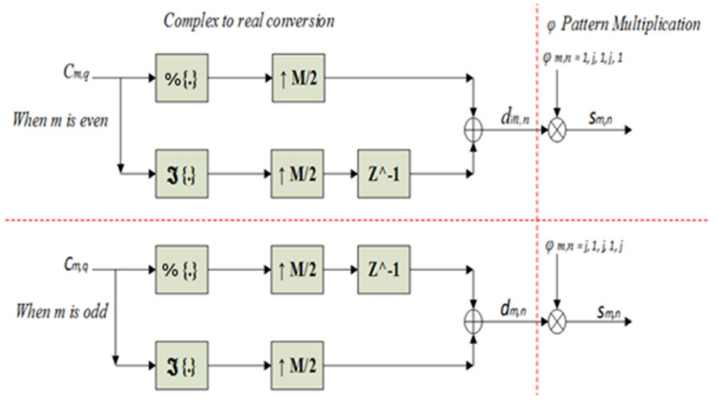

Fig. 1. OQAM pre-processing in the FBMC transmitter

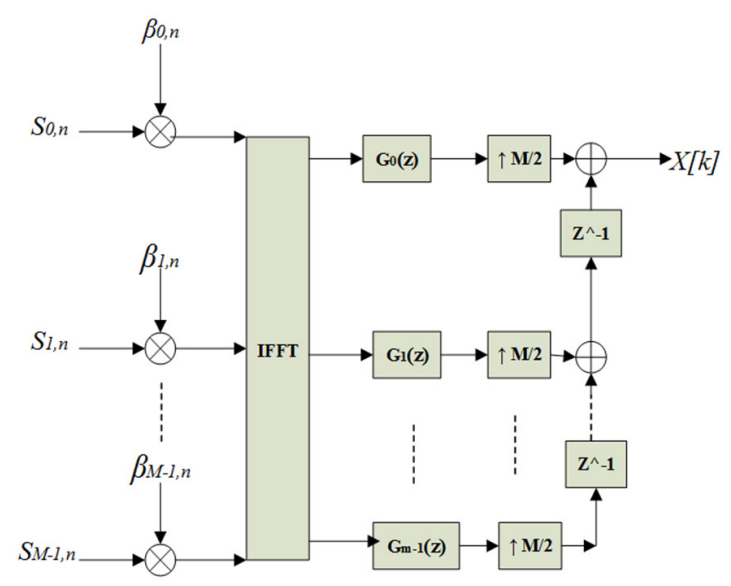

Fig. 2. SFB implementation in the FBMC transmitter

A discrete-time baseband FBMC-OQAM signal $(X[k])$ upsampled by a factor $M / 2$ is then obtained [21]:

$$
\begin{aligned}
& X[k]=\mathcal{G}\left\{\widehat{\mathrm{S}}_{0, \mathrm{n}}, \widehat{\mathrm{S}}_{1, \mathrm{n}}, \ldots \ldots \ldots \ldots \ldots, \widehat{\mathrm{S}}_{\mathrm{M}-1, \mathrm{n}}\right\} \\
& X[k]=\sum_{m=0}^{2 M-1} \sum_{n=0}^{N-1} d_{m, n} g\left[k-m \frac{T N}{2}\right] e^{j \frac{2 \pi}{T N} n k} e^{j \beta_{m, n}}
\end{aligned}
$$

where $\mathcal{G}\{$.$\} is the FBMC-OQAM modulation function, d_{m, n}$ are OQAM processed from $C_{m, q}$ vectors and $\beta_{m, n}$ is the phase term equal to $\frac{\pi}{2}(m+n)-\pi m$. The Physical Layer for Dynamic Spectrum Access and cognitive radio (PHYDYAS) prototype filter introduced in [22, 23] with overlapping factor $K=4$ is considered here as the pulse shaping filter in the FBMC-OQAM system because of its optimal localization characteristics of the signal in both time and frequency domains.

\section{B. CFR-Based Sequential Asymmetric Superposition Windowing Algorithm}

A major challenge of dealing with multicarrier systems such as FBMC-OQAM is the resulting high peak values. This mainly occurs because each subcarrier is usually modulated and filtered individually which results in more instantaneous power than the average power. The peak-to-average power ratio (PAPR) of the discrete-time FBMC-OQAM signal is [24]:

$$
\operatorname{PAPR}(X[k])=\frac{\max _{0 \leq k \leq K-1}\left(|X(k)|^{2}\right)}{E\left\{|X(k)|^{2}\right\}}
$$

where $E\{$.$\} is the expectation value.$

Here, the advantage of the sequential asymmetric superposition windowing (SASW) algorithm is explored because of its application in both single and multicarrier signals. At first, a window length is specified and when the peaks are detected in the window, they are grouped into blocks, and their locations are then indexed. In order to minimize the over-attenuation introduced by the unnecessary window superpositioning, an overall windowing function for each block is constructed in an iterative manner to deal with the dynamic fluctuation of the peaks. The window segment for the grouped peaks is then smoothened by the addition of all the asymmetric peak windows in the block. The sequential asymmetric superposition windowing (SASW) algorithm is applied to the peaks in each block, where only the peaks with large values take part in the decision making of the scaling function. The crest factor reduction (CFR) based on sequential asymmetric superposition windowing is given by [12]:

$$
X_{C F R}[k]=1-\sum_{i=-\infty}^{\infty} c_{s}[i]\left\{U_{l s}[k-k i]+U_{r s}[k-k i]\right\}
$$

where $U_{l s}[k]$ and $U_{r s}[k]$ are the left and right side of the window functions respectively with different window lengths, $c_{S}[i]$ is the new weighting index factor for those concealed peaks indexed by $i$ as $c_{s}[i]=0$.

\section{Digital Pre-Distortion in Wireless Systems Fundamentals}

The basic concept of the DPD technique is illustrated in Figure 3. A transfer function of a nonlinear PA is shown with $X_{D P D}[k]$ as the input and $y(k)$ as the output. If the DPD model manipulates the signal with a proper inverse transfer function such as shown on the transfer function with $X[k]$ as input and $X_{D P D}[k]$ as output, the final output $y(k)$ of the PA will be a linear signal with respect to the original input $X[k]$. Unlike circuit-level nonlinearity compensation, digital pre-distortion uses the black-box-based behavioral modeling procedures to describe and invert the input signal before the PA [25]. Under this concept, only the PA input and output relationship are considered in the nonlinearity compensation process. This significantly relieves the burden of analogue circuit design and debugging.

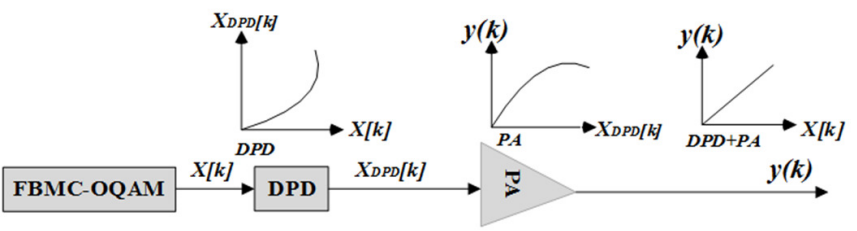

Fig. 3. Basic concept of digital pre-distortion

\section{Digital Pre-Distortion Model Description}

The power amplifier is an essential component of the transmitter. However, its operation near the saturation zone creates nonlinear distortion to the input signals which result in spectrum re-growth. Therefore, there is a need to compensate these nonlinearities in order to maintain the spectrum of the input signal within the specified band of interest. The 
nonlinearity of the PA is usually dynamic due to its build-up components, aging, and other environmental factors. Therefore, an adaptive identification of the dynamic nonlinear behavior of the PA is essential. To compensate for this nonlinear and the dynamic distortion in the PAs, adaptive DPD models based on indirect learning architecture (ILA) with memory polynomial (MP) are used because of their simplicity [10]. This concept is illustrated in Figure 4, where the adaptive DPD model is applied to adjust the signal sample $X[k]$ in the digital domain to compensate for the nonlinear behavior of the PA output $y(k)$ in the analogue domain. The PA output is monitored by an observation path and converted to the digital domain where the input signal $X_{D P D}[k]$ to the PA and the feedback signal $Z[k]$ from the $\mathrm{PA}$ output are compared and an adaptive algorithm is implemented where the DPD coefficients in the pre-distorter block are updated accordingly. The overall idea of using the adaptive DPD is to adjust the FBMC-OQAM signal $X[k]$ in such a way as to minimize the distortion introduced by the nonlinear PA in a dynamic manner.

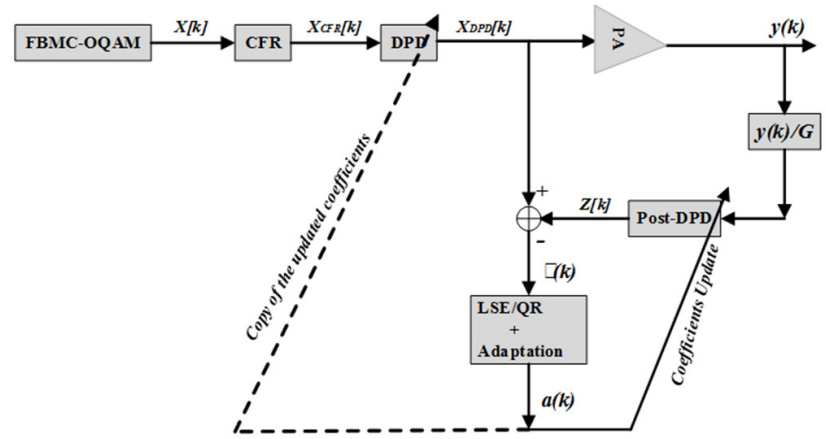

Fig. 4. ILA for an adaptive digital pre-distortion model extraction

\section{E. Digital Pre-Distortion Authentication Procedures}

The DPD can be carried out in multi-step procedures where the adjacent-channel-power (ACP) assessment of the transmitter is verified. Due to the dynamic nonlinear distortion and memory effects of the nonlinear PA, the DPD parameters are trained for several iterations. However, this requires a robust testing platform in order to evaluate the DPD performance properly. This work only considers the simulation-based platform. DPD algorithms are intrinsically mathematical and need to be performed on a processor with high computational ability, therefore, a simulation-based environment, known as SystemVue2018 has been used as the modeling and verification platform. The modeling procedures are:

Step 1 Baseband Source: Generating a digital baseband data sequence with required sampling rate such as FBMCOQAM signal $(X[k])$.

Step 2 Crest Factor Reduction: Reducing the peak of the FBMC-OQAM baseband signal in order to minimize clipping during amplification.

Step 3 Signal Pre-distortion: The original digital baseband signal is pre-distorted using an inverse transfer function model of the PA. This compensates the nonlinearity distortion effects of the PA.

Step 4 Signal Up-Conversion: Up-converting the digital baseband signal to the required analogue frequency, with appropriate signal power in order to drive the PA.

Step 5 Signal Amplification: A Power Amplifier is employed to amplify the signal before transmission to compensate for the attenuations that may be encountered by the signal in the free space propagation.

Step 6 Feedback Signal Attainment: The output of the nonlinear PA is down-converted to the baseband signal, and then the baseband analogue signal is captured with an ADC.

Step 7 DPD Parameter Extraction and Updating: The DPD parameters are calculated and then the coefficients are updated in the digital pre-distorter.

Step 8 Time Alignment: To obtain matched PA input and output, the original input and output signals of the PA are captured and aligned in the time domain so that the sample delay introduced by the feedback path can be characterized and compensated with no system deadlock.

Step 9 Performance Assessment: A spectrum analyzer is used to assess the frequency-domain nonlinearity compensation performance such as the ACLR or capturing the signal gain to assess the time-domain performance such as the EVM.

These procedures are modeled in SystemVue2018-based platform as illustrated in Figure 5.

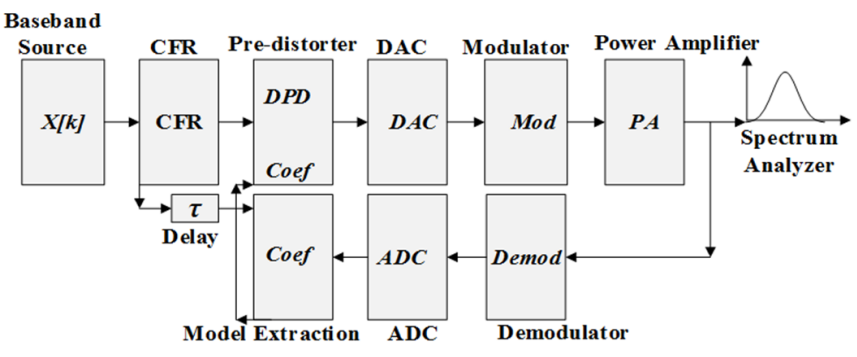

Fig. 5. A Simulation-based DPD procedure

\section{BEHAVIOR MODELING OF THE DIGITAL PRE-DISTORTION} SYSTEM

\section{A. Memory Polynomial Model}

The MP model has been considered as a special case of the Volterra model due to its elegancy and simple structure for incorporating memory effects into the static nonlinear polynomial model and has been extensively used in a variety of applications, especially in the linearization of power amplifiers with memory [10]. The nonlinearities with memory effects can be generally described as the sum of the outputs from the polynomial functions and can be written as [11]:

$$
X_{D P D}[k]=\sum_{p=1}^{P} \sum_{q=0}^{Q} a_{p, q} X[k-q]|X[k-q]|^{p-1}(5)
$$

where $P$ and $Q$ are the nonlinearity order and memory depth of the MP-based model respectively and $a_{p, q}$ are the coefficients of the model. 
The output of the nonlinear PA $y(k)$ is a function of the DPD signal $X_{D P D}[k]$ and is given by:

$$
y(k)=\sum_{p=1}^{P} \sum_{q=0}^{Q} a_{p, q} X_{D P D}(k-q)\left|X_{D P D}(k-q)\right|^{p-1}(6)
$$

In the indirect learning architecture DPD (Post-DPD Figure 4), the nonlinear function of the PA is inverted in order to identify the nonlinearity behavior of the PA which acts as a feedback signal, essentially acting as a post-distorter on the PA output signals. Since the nonlinearity behavior of the PA is modeled by DPD using MP, the pre-distortion of the nonlinear $\mathrm{PA}$ is functionally the same as the post-distortion. The input to the Post-DPD block is the direct inversion of the nonlinear PA transfer function and is given as:

$$
Z[k]=\sum_{p=1}^{P} \sum_{q=0}^{Q} a_{p, q} y(k-q)|y(k-q)|^{p-1}
$$

where the parameters $P, Q$, and $a_{p, q}$ are the same as in the predistortion model.

\section{B. Coefficients Estimation and Adaptation Algorithm}

FBMC-OQAM is the most demanding modulation scheme for the next generations of wireless communication industry. A relatively large number of samples have to be allocated to it to identify the small number of coefficients for the DPD algorithm. Therefore, the LSE with QR decomposition (LSE/QR) algorithm is used to estimate the coefficients of the model due to the over-determined problem experienced on the signal because of the large number of the samples used. The LSE is more commonly used because of its easy implementation and satisfactory performance [17].

\section{1) Coefficients Estimation}

The coefficients $a_{p, q}$ in (7) can be solved by using the LSE by defining a new signal variable as in [17]. The output of the pre-distorter training block (Post-DPD) of Figure 4 with the gain of the amplifier $G$ set to unit can be solve using the LSE solution as given in [17] where the coefficients of the predistorter training block (Post-DPD) become:

$$
\hat{a}=\left(U^{H} U\right)^{-1} U^{H} \hat{Z}
$$

The matrix $U$ is ill-conditioned even if the signal is scaled and normalized at the range of the values of the polynomial orders. Using the $Q R$ decomposition method, the matrix $U$ can be factorized into:

$$
U=Q R
$$

where the matrix $Q$ is $m \times n$ with orthonormal columns and $R$ is an invertible $n \times n$ upper triangular matrix. The orthonormal matrix $Q$ preserves the norm or distance in any transformation. The $Q R$ decomposition rotates the matrix $U$ until the point where the set of linear equations can be solved by backsubstitution in the matrix $R$, since $Q$ is orthonormal, i.e.:

$$
Q^{H} Q=I
$$

Then:

$$
Q^{H} \hat{z}=Q^{H} Q R \hat{a}=R \hat{a}
$$

Since $R$ is invertible, the estimate for the coefficients becomes:

$$
\hat{a}=R^{-1} Q^{H} \hat{Z}
$$

where $\left[\bullet{ }^{\mathrm{H}}\right.$ stands for complex conjugate transpose.

Equation (12) is relatively straightforward to solve, and since $R$ is triangular, the coefficients can be found by backsubstitution in the vector $Q^{H} \hat{Z}$. This procedure avoids the inversion of the $U^{H} U$ in the normal equation (8) and the associated numerical instabilities. This algorithm is in-built in the SystemVue2018 software and it is generally robust in dealing with ill-conditioned and rank-deficient matrices. The coefficients of the pre-distorter obtained off-line through (12) are copied to the pre-distorter on the feed-forward path as the initial coefficients. Because of the dynamic nature of the PA nonlinearity stated above, the coefficients can be updated adaptively as demonstrated in Figure 4.

\section{2) Coefficients Adaptation}

The pre-distortion coefficients adaptation is quite straightforward for indirect learning architecture. Considering the nonlinear memory polynomial model of the DPD as in (5), the pre-distorted input signal to the PA $\left(X_{D P D}[k]\right)$ is compared to the post-distorted output signal $(Z[k])$ from the PA. The absolute error signal $(\varepsilon)$ at any sample instant $k$ is then obtained by the equation given in [10]. Then, the estimate of the coefficients error $(\Delta a)$ can be minimized by taking the difference between the error term and the post-distortion update in the least square manner as given in [10]. The coefficient update relation can be expressed starting from the LSE expression by describing the coefficient estimation from the output of the post-distorted signal $(Z[k])$ and the pre-distorted $\operatorname{signal}\left(X_{D P D}[k]\right)$ as:

$$
X_{D P D(i)}=Z \cdot a
$$

In block matrix form:

$$
\widehat{a_{\imath}}=\left(Z_{i}^{H} Z_{i}\right)^{-1} Z_{i}^{H} \cdot X_{D P D i}
$$

Then the error term for the estimation block uses the previously-calculated coefficients and is written as:

$$
\varepsilon=Z_{i+1} \cdot \widehat{a}_{\imath}-X_{D P D_{i+1}}
$$

Expanding (15) and upon simplification, we get:

$$
\widehat{a}_{\imath}-\hat{a}_{i+1}=-\Delta a
$$

Therefore, the error expression for the pre-distorter coefficients becomes:

$$
\varepsilon=-Z_{i+1} \cdot \Delta a
$$

This coefficient error is used to update the pre-distorter coefficients through:

$$
\hat{a}_{i+1}=\widehat{a}_{l}-\mu \cdot \Delta a
$$

where the parameter $\mu$ is used to speed up or stabilize the convergences of the pre-distorted signal and it depends on the number of the coefficients used.

\section{SimUlation RESUlTS AND PERFORMANCE ANALYSIS}

This section presents the simulation results of the performance of the FBMC-OQAM signal in terms of the error 
vector magnitude (EVM) and adjacent channel leakage ratio (ACLR) when subjected to the nonlinear power amplifier. These performances are evaluated when the nonlinearities are compensated with concurrent application of CFR and DPD. The 16QAM mapping method has been used for the FBMCOQAM system because it is less susceptive to noise and data errors. An FBMC-OQAM signal with PHYDYAS prototype filter with overlapping factor $K=4$ is employed. The simulation parameters for the FBMC-OQAM signal are based on the 3 GPP release 13 to 15 and beyond [26] and the nonlinear power amplifier parameters settings are shown in Table I and II respectively.

TABLE I. FBMC-OQAM SIMULATION PARAMETERS

\begin{tabular}{|c|c|}
\hline Parameters & Values \\
\hline Carrier frequency & $6 \mathrm{GHz}$ \\
\hline Sampling rate with oversampling & $320 \mathrm{MHz}$ \\
\hline Sampling rate without oversampling & $20 \mathrm{MHz}$ \\
\hline Oversampling ratio & 4 \\
\hline Modulation type & $16 \mathrm{QAM}$ \\
\hline Number of subcarriers & 512 Subcarriers \\
\hline IFFT length & 2048 \\
\hline Filter overlapping factor & 4 \\
\hline Filter bank structure & Polyphase network IFFT \\
\hline
\end{tabular}

TABLE II. NONLINEAR POWER AMPLIFIED PARAMETER SETTINGS

\begin{tabular}{|c|c|}
\hline Parameters & Settings \\
\hline Amplifier gain & $1 \mathrm{~dB}$ \\
\hline Output 1dB gain compression power & $0.01 \mathrm{~W}$ \\
\hline Output third order intercept power & $0.1 \mathrm{~W}$ \\
\hline Saturation power & $0.032 \mathrm{~W}$ \\
\hline Gain compression at saturation & $3 \mathrm{~dB}$ \\
\hline Reference impedance & $50 \Omega$ \\
\hline
\end{tabular}

In the crest factor reduction (CFR), a peak windowing based on sequential asymmetric superposition windowing (SASW) with maximum Kaiser window length of 500 to enable the smooth transition on the window edge is used. A Kaiser window adjustment parameter $(\beta)$ equal to 15 to determine the rolling-off window's edge and maximum iteration time equal to 20 was considered due to its steady convergence level. A block size for each CFR operation of 1000 and a target PAPR value of $6 \mathrm{~dB}$ were considered. The CFR technique performance results are shown in Figure 6 where the peak value of the original FBMC-OQAM signal has been reduced by about $1.5 \mathrm{~dB}$. For the DPD technique, the memory polynomial model was used with nonlinear order $P=7$ and a memory depth of $Q=3$, which only consider odd-order polynomials due to the odd-order intermodulation product characteristics of the nonlinear PA transfer function. Figure 7(a) shows the power spectrum density (PSD) plot of the original FBMC-OQAM signal. The amplified version of the FBMC-OQAM signal without DPD is labeled as (b), and (c) is the DPD response. Although the DPD responded by suppressing a significant amount of the spectral re-growth, less gain is experienced after the nonlinearity compensation with the DPD. This is because of the high peak characteristics of the FBMC-OQAM signal and the nonlinearity behavior of the PA which exhibited high spectral re-growth after the amplification of the FBMC-OQAM signal.

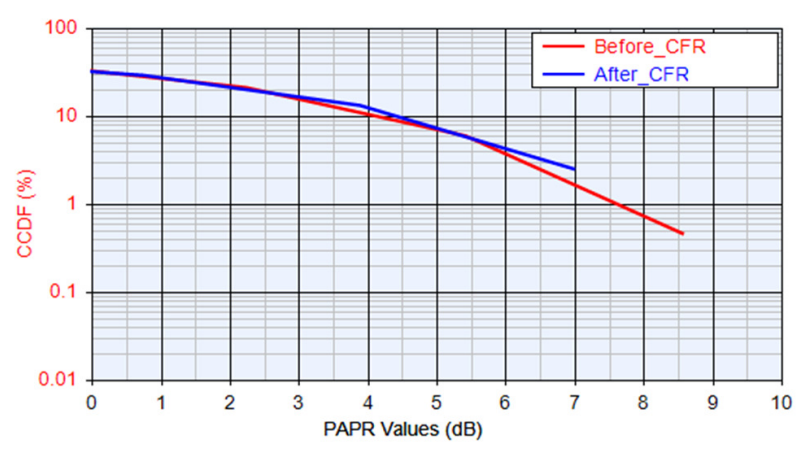

Fig. 6. CCDF plot of the performance of the CFR technique

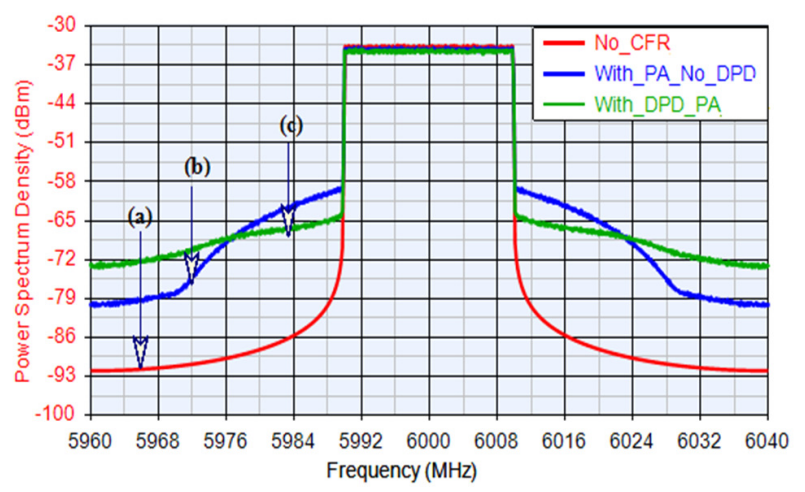

Fig. 7. PSD of (a) original FBMC-OQAM signal, (b) PA output without DPD, (c) PA output with DPD

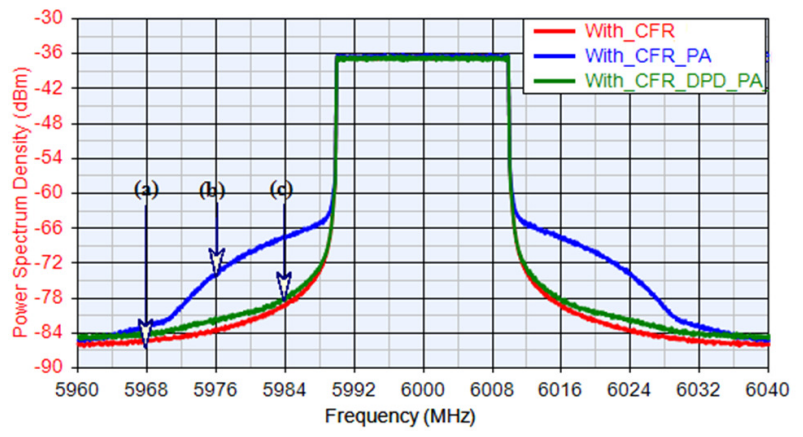

Fig. 8. PSD of (a) reduced crest factor of the FBMC-OQQAM signal, (b) PA output, (c) PA output with combined CFR and DPD

It can be observed in Figure 8 that using CFR simultaneously with DPD can significantly reduce the spectral re-growth by about $-45.74 \mathrm{~dB}$ adjacent channel leakage suppression. The error vector magnitude (EVM) measure is found to be about $7.12 \%(-22.95 \mathrm{~dB})$ which is clearly reaching the target of the standard EVM value for wideband signals which was standardized to be $8 \%(-22 \mathrm{~dB})$ by $3 \mathrm{GPP}$.

\section{CONCLUSION}

Spectral re-growth suppression of the FBMC-OQAM signal under the nonlinear behavior of a power amplifier has been studied in this paper. Regarding the CFR of the FBMC-OQAM signal, a peak windowing technique with sequential asymmetric superposition windowing (SASW) algorithm has been proposed, which showed consistency in reducing the large 
peak values of the FBMC-OQAM signal by about $1.5 \mathrm{~dB}$. This leads to an increase in the average input power of the FBMCOQAM signal. Regarding nonlinearity compensation, memory polynomial based DPD with LSE along with QR decomposition (LSE/QR) which utilized the indirect learning architecture (ILA-DPD) have been employed. The CFR and DPD techniques have been jointly applied to mitigate the issues of both high peak and nonlinearities experienced on the FBMC-OQAM signals. The application of both CFR and DPD significantly reduced the spectral re-growth which resulted from the nonlinear behavior of the PA by about $-45.74 \mathrm{~dB}$ adjacent channel leakage ratio (ACLR) suppression. The error vector magnitude (EVM) measure was found to be about $7.12 \%(-22.95 \mathrm{~dB})$ which is less than the EVM value for wideband signals standardized by $3 \mathrm{GPP}$ to be $8 \%(-22 \mathrm{~dB})$. In this paper, much emphasis has been given to the FBMCOQAM waveform. Nevertheless, it would be of equal interest to study other waveforms and compare them with FBMCOQAM under the same proposed techniques, in the $5 \mathrm{G}$ context.

\section{REFERENCES}

[1] W. Jiang, T. Kaiser, "From OFDM to FBMC: Principles and comparisons", in: Signal Processing for 5G: Algorithms and Implementations, John Wiley \& Sons, 2016

[2] S. Patil, S. Patil, U. Kolekar, "Implementation of 5G using OFDM and FBMC (filter bank multicarrier) /OQAM (offset quadrature amplitude modulation)", International Journal of Innovative Science, Engineering \& Technology, Vol. 5, No. 1, pp. 11-15, 2018

[3] H. Zhang, D. L. Ruyet, D. Roviras, Y. Medjahdi, H. Sun, "Spectral Efficiency Comparison of OFDM/FBMC for Uplink Cognitive Radio Networks", EURASIP Journal on Advances in Signal Processing, Vol. 2010, Article ID 621808, 2010

[4] R. Gerzaguet, N. Bartzoudis, L. G. Baltar, V. Berg, J. B. Dore, D. Ktenas, O. F. Bach, X. Mestre, M. Payaro, M. Farber, K. Roth, "The 5G candidate waveform race: a comparison of complexity and performance", EURASIP Journal on Advances on Wireless Communications and Networking, Vol. 1, No. 1, pp. 1-14, 2017

[5] M. Renfors, X. Mestre, E. Kofidis, F. Bader, Orthogonal Waveforms and Filter Banks for Future Communication Systems, First Edition, Academic Press, 2017

[6] R. Nissel, S. Schwarz, M. Rupp, "Filter bank multicarrier modulation schemes for future mobile communications", IEEE Journal on Selected Areas in Communications, Vol. 35, No. 8, pp. 1768-1782, 2017

[7] M. Azhar, A. Shabbir, "5G networks: Challenges and techniques for energy efficiency", Engineering, Technology \& Applied Science Research, Vol. 8, No. 2, pp. 2864-2868, 2018

[8] A. Shabbir, H. R. Khan, S. A. Ali, S. Rizvi, "Design and performance analysis of multi-tier heterogeneous network through coverage, throughput and energy efficiency", Engineering, Technology \& Applied Science Research, Vol. 7, No. 6, pp. 2345-2350, 2017

[9] Y. Rahmatallah, S. Mohan, "Peak-to-average power ratio reduction in OFDM systems: A survey and taxonomy", IEEE Communications Surveys and Tutorials, Vol. 15, No. 4, pp. 1567-1592, 2013

[10] J. Wood, Behavioral Modeling and Linearization of RF Power Amplifiers, First Edition, Artech House, 2014

[11] Z. He, W. Ye, S. Feng, "Digital predistortion of power amplifiers based on compound memory", IEICE Electronic Express, Vol. 10, No. 21, pp. $1-5,2013$

[12] M. V. D. Nair, R. Giofre, P. Colantonio, F. Giannini, "Sequential Asymmetric Superposition Windowing for Crest Factor Reduction and its Effects on Doherty Power Amplifier", Integrated Nonlinear Microwave and Millimetre-wave Circuits Workshop, Taormina, Italy, October 1-2, 2015

[13] M. V. D. Nair, R. Giofre, P. Colantonio, F. Giannini, "Effects of Digital Predistortion and Crest Factor Reduction Techniques on Efficiency and
Linearity Trade-off in Class AB GaN-PA", 10th European Microwave Integrated Circuits Conference, Paris, France, September 7-8, 2015

[14] F. M. Ghannouchi, O. Hammi, M. Helaoui, Behavioral Modeling and Predistortion of Wideband Wireless Transmitters, First Edition, John Wiley \& Sons, 2015

[15] R. Zayani, Y. Medjahdi, H. Bouhadda, H. Shaiek, D. Roviras, R. Bouallegue, "Adaptive Predistortion Techniques for Non-Linearly Amplified FBMC-OQAM Signals", IEEE 79th Vehicular Technology Conference, Seoul, South Korea, May 18-19, 2014

[16] M. Sajedin, A. Ghorbani, H. R. A. Dava, "Nonlinearity compensation for high power amplifiers based on look-up table method for OFDM transmitters", International Journal of Advanced Computer Science and Information Technolog, Vol. 3, No. 4, pp. 354-367, 2014

[17] W. Gao, Linearization Techniques for RF Power Amplifiers, Springer, 2017

[18] H. D. Rodrigues, T. C. Pimenta, R. A. A. D. Souza, L. L. Mendes, "Orthogonal scalar feedback digital pre-distortion linearization", IEEE Transactions on Broadcasting, Vol. 64, No. 2, pp. 319-330, 2018

[19] V. Vasconcellos, G. C. Ornelas, A. N. Barreto, "Performance of 5G Candidate Waveforms with Non-Linear Power Amplifiers", IEEE 9th Latin-American Conference on Communications, Guatemala City, Guatemala, November 8-10, 2017

[20] S. S. K. C. Bulusu, H. Shaiek, D. Roviras, "PA Linearization of FBMCOQAM Signals with Overlapped Recursive Error Correcting Predistortion", International Symposium on Wireless Communication Systems, Poznan, Poland, September 20-23, 2016

[21] T. Jiang, D. Chen, C. Ni, D. Qu, OQAM/FBMC for Future Wireless Communications Principles Technologies and Applications, First Edition, Academic Press, 2018

[22] M. Bellanger, "FBMC physical layer: A primer", European Project, Vol. 1, No. 1, pp. 1-31, 2010

[23] A. Sahin, I. Guvenc, H. Arslan, "A survey on multicarrier communications: Prototype filters, lattice structures, and implementation aspects", IEEE Communications Surveys and Tutorials, Vol. 16, No. 3, pp. 1312-1338, 2014

[24] H. Wang, "A Hybrid PAPR Reduction Method Based on SLM and Multi-Data Block PTS for FBMC/OQAM Systems", Information, Vol. 9, Article ID 246, 2018

[25] L. Guan, A. Zhu, "Green communications: Digital predistortion for wideband RF power amplifier", IEEE Microwave Magazine, Vol. 15, No. 7, pp. 84-99, 2014

[26] 5G Americas, Wireless Technology Evolution Towards 5G: 3GPP Release 13 to Release 15 and Beyond, 5G Americas, 2017 